obese patients have increased morbidity in terms of septic complications following liver transplantation, with consequent increased length of intensive care and hospital stay. Identifying these patients early and introduction of measures to reduce BMI should be considered to improve outcomes following liver transplantation.

Competing interests None declared.

\section{OC-059 AN EXPERIMENTAL STUDY TO DETERMINE THE PATHOGENESIS OF OXALIPLATIN INDUCED SINUSOIDAL OBSTRUCTION SYNDROME}

doi:10.1136/gutjnl-2012-302514a.59

\begin{abstract}
${ }^{1,2} \mathrm{~S}$ Robinson, ${ }^{*}{ }^{2} \mathrm{~J}$ Mann, ${ }^{1} \mathrm{D}$ Manas, ${ }^{2} \mathrm{~A}$ Burt, ${ }^{2} \mathrm{D}$ Mann, ${ }^{1} \mathrm{~S}$ White. ${ }^{1} H P B$ \& Transplant Surgery, Freeman Hospital, Newcastle Upon Tyne, UK; ${ }^{2}$ Institute of Cellular Medicine, Newcastle University, Newcastle Upon Tyne, UK
\end{abstract}

Introduction Sinusoidal obstruction syndrome (SOS) following Oxaliplatin based chemotherapy is a cause for major concern when undertaking liver resection for colorectal liver metastases. To date no relevant experimental models of Oxaliplatin induced SOS have been described. The aim of this project was to establish such a model which could be utilised to identify potential therapeutic strategies to prevent the development of SOS.

Methods C57Bl/ 6 mice were treated with intra-peritoneal FOLFOX $(n=10)$, or vehicle $(n=10)$, weekly for 5 weeks and culled 1 week following final treatment. Representative biopsies of the liver and spleen were fixed in formalin and paraffin embedded for histological analysis. RNA and protein were extracted from snap frozen biopsies of the liver and subject to biochemical, analysis by qRT-PCR and western blot respectively, for markers of matrix remodelling, vascular dysfunction/endothelial damage, DNA damage and cellular proliferation. Serum was separated from whole blood and markers of liver injury (ie, ALT, AST and Alk Phos) were also measured. Statistical significance was assessed with Mann-Whitney U Test.

Results FOLFOX treatment was associated with the development of sinusoidal dilatation and peri-venular hepatocyte atrophy on H\&E stained sections of the liver in keeping with SOS. This was associated with an elevated serum ALT and AST $(p<0.05)$. Immunohistochemistry for $\gamma \mathrm{H} 2 \mathrm{AX}$ demonstrated the presence of DNA damage in the sinusoidal endothelium. In the liver of FOLFOX treated animals there was up-regulation of key genes associated with matrix remodelling such as MMP9 $(p<0.001)$, MMP2 $(p<0.001)$, ProCollagen I $(p<0.001)$ and TGF $\beta(p<0.001)$. There was evidence of endothelial damage and a subsequent pro-thrombotic state with upregulation of PAI-1 $(p<0.001)$, vWF $(p<0.01)$ and Factor $X(p<0.001)$. Conclusion We have developed the first reproducible model of chemotherapy induced SOS that reflects the pathogenesis of this disease process in patients. Through analysis of this model we have gained insights into the molecular changes that underpin the development of SOS and are now able to test potential therapeutic strategies to prevent it.

Competing interests None declared.

\section{OC-060 SURVIVAL AFTER DOWNSTAGING CHEMOTHERAPY FOR INITIALLY UN-RESECTABLE COLORECTAL LIVER METASTASES: EXPERIENCE FROM A UK RESECTION CENTRE}

doi:10.1136/gutjnl-2012-302514a.60

${ }^{1} \mathrm{G}$ R Irving, * 1 J P Tiernan, ' C D Briggs, 'M Peterson, ${ }^{2} \mathrm{C}$ Cameron. ${ }^{1} \mathrm{HPB}$ surgery, Sheffield Teaching Hospitals, Sheffield, UK; ${ }^{2} H P B$ surgery, Queens Medical Centre, Nottingham, UK

Introduction Liver metastases occur in $\sim 50 \%$ of patients with colorectal cancer. Only $20 \%$ of patients present with disease that is suitable for resection, the only hope of cure. In selected patients, chemotherapy may downstage inoperable liver-limited disease such that potentially curative resection becomes feasible.

Methods All patients referred to the regional Multi-Disciplinary Team (October 2001-June 2008) were considered for downstaging chemotherapy if they had inoperable liver-limited disease and were fit enough for resection. Two-weekly FOLFOX chemotherapy was administered and response assessed by 3 monthly CT scan. Disease having a partial response but remaining unresectable received further chemotherapy and reassessment by CT. Patients in whom R0 resection was thought feasible were offered surgery. Morbidity and mortality data were collected. Mortality was cross-referenced with the Cancer Registry. Overall (OS), post-operative (POS) and disease free survival (DFS) were calculated using SPSS (medians/ range/Kaplan-Meier survival curves). OS was calculated from the onset of chemotherapy and based on intention to treat. Additional univariate analysis has been performed.

Results 104 patients commenced downstaging chemotherapy (median six cycles): one died after the 3rd cycle (cardiac), 28 had no response, 56 had a degree of response and 19 had near complete regression. Eventually, 56 patients remained unresectable and 47 had a disease response deemed resectable and were offered surgery. Of these 47 patients, seven declined or became unfit for surgery and 40 proceeded to an operation, of which 36 underwent resection. In four, liver resection was abandoned due to additional disease found at surgery. Peri-operative morbidity was $63 \%$ and 30 -day mortality was zero. Mortality of patients receiving only chemotherapy was $100 \%$ and median OS 14 months (range 3-64 months) compared to a median OS for the 40 patients undergoing laparotomy of 39 months (10-98) with an estimated 20\% 5-year survival rate. The 10 surviving patients $(25 \%)$ have been followed up for a median of 63.5 months (36-90). 7/36 patients (19.4\%) remain disease free with median OS 85 months (40-98). $29 / 36$ patients (80.6\%) have recurred, all within 24 months of surgery (median DFS 7 months) but with median OS 35 months (10-73) and 4/29 (14\%) surviving $>5$ years.

Conclusion Liver resection after downstaging chemotherapy is safe, feasible and improves median survival. $80 \%$ of patients had recurring disease, all within 2 years, however a significant survival benefit occurred in this group compared to patients who could not be offered surgery. The proportion of patients remaining disease free $(19.4 \%)$ is lower than would be expected in a group with initially resectable disease.

Competing interests None declared.

\section{OC-061 GASTRIC ELECTRICAL STIMULATION FOR INTRACTABLE GASTROPARESIS}

doi:10.1136/gutjnl-2012-302514a.61

${ }^{1} \mathrm{~A}$ I Sarela, ${ }^{*} \mathrm{~S}$ Dexter, ${ }^{2} \mathrm{~F}$ Choudhury, ${ }^{3} \mathrm{M}$ Denyer. ${ }^{1}$ Upper Gl Surgery, St James's University Hospital, Leeds, UK; ${ }^{2}$ Nuclear Medicine, St James's University Hospital, Leeds, UK; ${ }^{3}$ Department of Gastroenterology, St James's University Hospital, Leeds, UK

Introduction Severe, drug-unresponsive gastroparesis is a debilitating condition. Treatment with gastric electrical stimulation using Enterra ${ }^{\circledR}$ was granted humanitarian device exemption status by the FDA in 2000. The largest case-series $(N=221)$ reports that $54 \%$ of patients had $>50 \%$ symptom-reduction with Enterra. ${ }^{1}$ We audited outcomes from Enterra therapy, with routine use of a trial of temporary stimulation to refine patient selection.

Methods Patients considered for Enterra during 2008-2011 were identified from a prospectively maintained database. Patients had been previously extensively investigated and treated for gastroparesis; $48 \%$ were referred by gastroenterologists and $37 \%$ by upper GI surgeons. Gastric emptying was assessed by scintigraphy in all 